Bull. Austral. Math. Soc.

Vol. 44 (1991) [451-460]

\title{
EXTENSIONS OF CERTAIN RESULTS IN WALSH-TYPE EQUICONVERGENCE
}

\author{
M.A. BOKHARI
}

Two sequences of rational functions are constructed from different expansions of $\left(t^{q n}-1\right)^{-1}$ and extensions of certain known results in the theory of Walsh-type equiconvergence are sought.

\section{INTRODUCTION}

Let $\pi$, denote the class of all polynomials of degree $\leqslant s$ over the field of complex numbers. For a given $\sigma>1$ and a fixed integer $m \geqslant-1$, let $\mathcal{R}_{n+m}$ denote the class of all rational functions of the form $p(z) /\left(z^{n}-\sigma^{n}\right), p(z) \in \pi_{n+m}$. We denote by $A_{\rho}$ $\rho>1$, the class of all functions analytic in $|z|<\rho$ but not in $|z| \leqslant \rho$, and consider the following minimisation problems:

(P1) [6] $\min _{r(z) \in \mathcal{R}_{n+m}} \int_{|z|=1}|f(z)-r(z)|^{2}|d z|$
(P2) [2] $\min _{r(z) \in \mathcal{R}_{n+m}} \sum_{k=0}^{q n-1}\left|f\left(\omega^{k}\right)-r\left(\omega^{k}\right)\right|^{2}$

where $q \geqslant 2$ and $\omega=\exp (2 \pi i q n)$.

It is known that for any $f \in A_{\rho}$, the elements $r_{n+m, n}(z, f)$ and $R_{n+m, n}^{*}(z, f)$ of $\mathcal{R}_{n+m}$ which respectively solves (P1) and (P2) are given by ((4.1) $\left.-(4.4),[2]\right)$

$$
r_{n+m, n}(z, f)=\frac{1}{2 \pi i} \int_{\Gamma} \frac{\left(t^{n}-\sigma^{n}\right) f(t)}{\left(z^{n}-\sigma^{n}\right)(t-z)} \sum_{j=1}^{3} A_{j}(t, z) d t
$$

and

$$
R_{n+m, n}^{*}(z, f)=\frac{1}{2 \pi i} \int_{\Gamma} \frac{f(t)}{\left(z^{n}-\sigma^{n}\right)(t-z)\left(t^{q^{n}}-1\right)} \sum_{j=1}^{3} A_{j}(t, z) B_{j}(t, \sigma) d t
$$

where $\Gamma$ is the circle $|t|=R, 1<R<\rho$ and

$$
\left\{\begin{array}{l}
A_{1}(t, z)=\frac{t^{m+1}-z^{m+1}}{t^{m+1}}, A_{2}(t, z)=\frac{z^{m+1}\left(t^{n-m-1}-z^{n-m-1}\right)}{t^{n}-\sigma^{-n}}, \\
A_{3}(t, z)=z^{n}\left(t^{m+1}-z^{m+1}\right) / t^{m+1}\left(t^{n}-\sigma^{-n}\right)
\end{array}\right.
$$

Received 3 December 1990

Copyright Clearance Centre, Inc. Serial-fee code: 0004-9729/91 \$A2.00+0.00. 
and

$$
\left\{\begin{array}{l}
B_{1}(t, \sigma)=\sigma^{-(q-2) n} B(t, \sigma)-t^{q n} \sigma^{n} \\
B_{2}(t, \sigma)=\left(t^{q n}-\sigma^{-q n}\right)\left(\sigma^{-n}-\sigma^{n}\right) /\left(1+\sigma^{-q n}\right), \\
B_{3}(t, \sigma)=\left(t^{n}-\sigma^{-n}\right)\left(t^{q n}-\sigma^{n} B(t, \sigma)\right),
\end{array}\right.
$$

with

$$
B(t, \sigma)=\frac{t^{n}\left(t^{(q-1) n}-\sigma^{-(q-1) n}\right)\left(1-\sigma^{-2 n}\right)}{\left(t^{n}-\sigma^{-n}\right)\left(1-\sigma^{-2(q-1) n}\right)}
$$

It has been shown in [2] that

$$
\begin{array}{r}
\lim _{n \rightarrow \infty}\left\{R_{n+m, n}^{*}(z, f)-r_{n+m, n}(z, f)\right\} \\
=0, \begin{cases}|z|<\rho^{1+q} & \text { if } \sigma \geqslant \rho^{1+q} \\
|z| \neq \sigma & \text { if } \sigma<\rho^{1+q}\end{cases}
\end{array}
$$

and that (1.5) extends the following theorem due to Rivilin [5]:

TheOREM A. Let $f(z):=\sum_{j=0}^{\infty} a_{j} z^{j} \in A_{\rho}, \rho>1$ and $S_{n-1}(z, f)=\sum_{j=0}^{n-1} a_{j} z^{j}$. Let $p_{n-1, q}(z, f), q \geqslant 2$, denote the polynomial of degree $n-1$ of least squares approximation to $f$ on the (nq)-th roots of unity. Then

$$
\lim _{n \rightarrow \infty}\left\{p_{n-1, q}(z, f)-S_{n-1}(z, f)\right\}=0, \quad \forall|z|<\rho^{1+q} .
$$

Another generalisation of (1.6) is that for any positive integer $\ell \geqslant 1$ and $f \in A_{\rho}$, we have

$$
\lim _{n \rightarrow \infty}\left\{p_{n-1, q}(z, f)-\sum_{k=0}^{\ell-1} S_{n-1, k}(z, f)\right\}=0, \quad \forall|z|<\rho^{1+\ell q}
$$

where $S_{n-1, k}(z, f)=\sum_{j=0}^{n-1} a_{j+k q} z^{j}, k=0,1, \ldots, \ell-1$.

It may be noted that a classical theorem of Walsh ([9] p.153) which deals with equiconvergence of certain sequences of polynomials is a special case for each of the results $(1.5)-(1.7)$. For further information on this topic we refer the interested reader to $[1,3,7]$.

Our object in this paper is to extend (1.5) in the spirit of (1.7). For this, we construct two different sequences of help rational functions which lead us to obtain a larger region of equiconvergence. These extensions are obtained from two different expansions of $\left(t^{q n}-1\right)^{-1}$. 


\section{EXTENSION I}

Our first extension is based on the following identity:

where

$$
\left(t^{q n}-1\right)^{-1}=\left[t^{q n}-\sigma^{-q n}-\left(1-\sigma^{-q n}\right)\right]^{-1}=\sum_{\nu=1}^{\infty} \widetilde{F}_{\nu}(t, \sigma)
$$

$$
\widetilde{F}_{\nu}(t, \sigma)=\frac{\left(1-\sigma^{-q n}\right)^{\nu-1}}{\left(t^{q n}-\sigma^{-q n}\right)^{\nu}}, \quad \nu=1,2, \ldots
$$

We define the rational functions

$$
\tilde{r}_{n+m, n}(z, f, \nu):=\sum_{j=0}^{n+m}\left(\tilde{c}_{j}(\nu) z^{j} / z^{n}-\sigma^{n}\right), \quad \nu=1,2,3, \ldots,
$$

where

$$
\tilde{c}_{j}(\nu):= \begin{cases}\frac{1}{2 \pi i} \int_{\Gamma} \frac{K_{1}(t, \sigma)}{t j+1} \widetilde{F}_{\nu}(t, \sigma) f(t) d t, & 0 \leqslant j \leqslant m, \\ \frac{1}{2 \pi i} \int_{\Gamma} \frac{K_{2}(t, \sigma) \widetilde{F}_{\nu}(t, \sigma)}{t^{m-n+j+2}\left(t^{n}-\sigma^{-n}\right)} f(t) d t, & m+1 \leqslant j \leqslant n-1, \\ \frac{1}{2 \pi i} \int_{\Gamma} \frac{K_{3}(t, \sigma) \widetilde{F}_{\nu}(t, \sigma)}{t^{j+1}\left(t^{n}-\sigma^{-n}\right)} f(t) d t, & n \leqslant j \leqslant n+m .\end{cases}
$$

with ([2], (4.6))

$$
K_{j}(t, \sigma)=B_{j}(t, \sigma)-\left(t^{q n}-1\right)\left(t^{n}-\sigma^{n}\right), \quad j=1,2,3
$$

where $B_{j}(t, \sigma)$ are given in (1.4). For $\nu=0$, we let

$$
\tilde{r}_{n+m, n}(z, f, 0):=r_{n+m, n}(z, f) .
$$

REMARK 1. From (2.1) we can rewrite

$$
\tilde{r}_{n+m, n}(z, f, \nu)=\frac{1}{z^{n}-\sigma^{n}}\left\{\sum_{j=0}^{m} \tilde{c}_{j}(\nu) z^{j}+\sum_{j=m+1}^{n-1} \tilde{c}(\nu) z^{j}+\sum_{j=n}^{n+m} \tilde{c}_{j}(\nu) z^{j}\right\},
$$

$(\nu=1,2,3, \ldots)$, so that using (1.3) we have

$$
\tilde{r}_{n+m, n}(z, f, \nu)=\frac{1}{2 \pi i} \int_{\Gamma} \frac{f(t) \tilde{F}_{\nu}(t, \sigma)}{\left(z^{n}-\sigma^{n}\right)(t-z)} \sum_{j=1}^{3} A_{j}(t, z) K_{j}(t, \sigma) d t .
$$

If we define

$$
\widetilde{W}_{n+m, n}(z, f, \ell):=\sum_{\nu=0}^{\ell-1} \widetilde{r}_{n+m, n}(z, f, \nu),
$$

we have the first extension of (1.5) (see [2], Theorem 2.1) given by: 
THEOREM 1. Let $m \geqslant-1, q \geqslant 2$ and $\ell \geqslant 1$ be three fixed integers and let $\sigma>1$. If $f \in A_{\rho}, 1<\rho<\infty$, then

$$
\lim _{n \rightarrow \infty}\left\{R_{n+m, n}^{*}(z, f)-\widetilde{W}_{n+m, n}(z, f, \ell)\right\}=0, \begin{cases}|z|<\rho^{\ell q+1} & \text { if } \sigma \geqslant \rho^{\ell q+1} \\ |z| \neq \sigma & \text { if } \sigma<\rho^{\ell q+1}\end{cases}
$$

the convergence being uniform and geometric on any compact subset of the regions defined above. Moreover, the result is sharp in the sense that for each $|z|=\rho^{1+\ell q}$, there is an $\hat{f} \in A_{\rho}$ for which (2.7) does not hold.

Proof: The difference in (2.7) can be written as

$$
\begin{aligned}
& R_{n+m, n}^{*}(z, f)-\widetilde{W}_{n+m, n}(z, f, \ell) \\
& \quad=R_{n+m, n}^{*}(z, f)-\widetilde{r}_{n+m, n}(z, f, 0)-\sum_{\nu=1}^{\ell-1} \widetilde{r}_{n+m, n}(z, f, \nu) .
\end{aligned}
$$

Applying (1.1), (1.2), (2.4) and (2.5) to the above relation we obtain

$$
\begin{aligned}
& R_{n+m, n}^{*}(z, f)-\widetilde{W}_{n+m, n}(z, f, \ell) \\
& \quad=\frac{1}{2 \pi i} \int_{\Gamma} \frac{\sum_{j=1}^{3} A_{j}(t, z) K_{j}(t, \sigma)}{\left(z^{n}-\sigma^{n}\right)(t-z)} \sum_{\nu=\ell}^{\infty} \widetilde{F}_{\nu}(t, \sigma) f(t) d t .
\end{aligned}
$$

Since $\sum_{\nu=\ell}^{\infty} \tilde{F}_{\nu}(t, \sigma)=\left(1-\sigma^{-q n}\right)^{\ell} /\left(\left(t^{q n}-\sigma^{-q n}\right)^{l-1}\left(t^{q n}-1\right)\right)$, we conclude (2.7) from (2.8) after some computation. As usual, the function $\hat{f}(z)=\left(z-p e^{i \theta}\right)^{-1}, 0 \leqslant \theta \leqslant 2 \pi$, shows that the result is sharp.

\section{Extension II}

Here we rearrange a double series in order to construct another sequence of help rational functions. First, note that for an absolutely convergent series $\sum_{s=1}^{\infty} \sum_{\lambda=1}^{\infty} g(s, \lambda)$ and a fixed integer $q \geqslant 1$, we have

$$
\begin{aligned}
\sum_{s=1}^{\infty} \sum_{\lambda=1}^{\infty} g(s, \lambda) & =\sum_{s=1}^{\infty} \sum_{j=1}^{\infty} \sum_{\lambda=1}^{q} g(s,(j-1) q+\lambda) \\
& =\sum_{\lambda=1}^{q} \sum_{s=1}^{\infty} \sum_{j=1}^{\infty} g(j,(s-j) q+\lambda)
\end{aligned}
$$


the last expression follows on writing the series $\sum_{j=1}^{\infty} \sum_{j=1}^{\infty} g(s,(j-1) q+\lambda)$, for each fixed $\lambda$, as shown below, and then on adding the terms along transverse diagonals as shown below

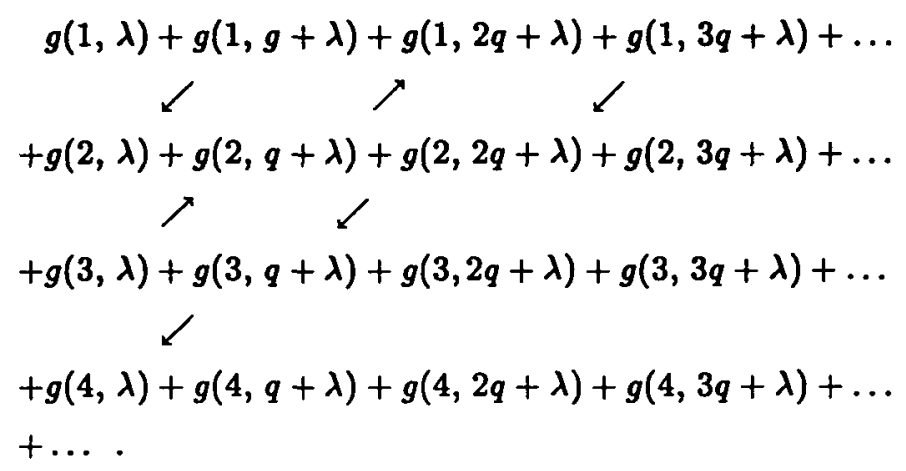

With this obervation, we have

Lemma 1. For $|t|>1$ and $\sigma>1$ the following identity holds:

$$
\left(t^{g n}-1\right)^{-1}=\sum_{\rho=1}^{\infty} \sum_{\lambda=1}^{q} F_{(,-1) q+\lambda}^{*}(t, \sigma)
$$

where

$$
\begin{aligned}
& F_{(o-1) q+\lambda}^{*}(t, \sigma) \\
& \quad=\sum_{j=1}^{\dot{1}}\left(\begin{array}{c}
(s-j) q^{2}+\lambda q+j-2 \\
j-1
\end{array}\right) \frac{\left(-\sigma^{-n}\right)^{j-1}}{\left(t^{n}-\sigma^{-n}\right)^{\left((-j) q^{2}+\lambda q+j-1\right.}} .
\end{aligned}
$$

Proof: It is easy to see the validity of the following expansion:

$$
\left(t^{q n}-1\right)^{-1}=\sum_{s=1}^{\infty} \sum_{\lambda=1}^{\infty}\left(\begin{array}{c}
\lambda q+s-2 \\
s-1
\end{array}\right) \frac{\left(-\sigma^{-n}\right)^{d-1}}{\left(t^{n}-\sigma^{-n}\right)^{\lambda q+c^{-1}}} .
$$

If we let $g(s, \lambda)=\left(\begin{array}{c}\lambda q+s-2 \\ s-1\end{array}\right)\left(\left(-\sigma^{-n}\right)^{s-1}\right) /\left(\left(t^{n}-\sigma^{-n}\right)^{\lambda q+s^{-1}}\right)$ in equation (3.1), then (3.2) follows immediately from (3.4) on observing that

$$
g(j,(s-j) q+\lambda)=\left(\begin{array}{c}
{[(s-j) q+\lambda] q+j-2} \\
j-1
\end{array}\right) \frac{\left(-\sigma^{-n}\right)^{j-1}}{\left(t^{n}-\sigma^{-n}\right)^{[(\cdot-j) q+\lambda] q+j-1}} .
$$


Now we define another sequence of help functions. Let

$$
r_{n+m, n}^{*}(z, f, \nu):=\sum_{j=0}^{m+n} c_{j}^{*}(\nu) z^{j} /\left(z^{n}-\sigma^{n}\right), \quad \nu=1,2,3, \ldots
$$

with

$$
c_{j}^{*}(\nu):= \begin{cases}\frac{1}{2 \pi i} \int_{\Gamma} \frac{K_{1}(t, \sigma)}{t+1} F_{\nu}^{*}(t, \sigma) f(t) d t, & 0 \leqslant j \leqslant m, \\ \frac{1}{2 \pi i} \int_{\Gamma} \frac{K_{2}(t, \sigma) F_{\nu}^{*}(t, \sigma)}{t^{m-n+j+2}\left(t^{n}-\sigma^{-n}\right)} f(t) d t, & m+1 \leqslant j \leqslant n-1, \\ \frac{1}{2 \pi i} \int_{\Gamma} \frac{K_{3}(t, \sigma) F_{\nu}^{*}(t, \sigma)}{t^{j+1}\left(t^{n}-\sigma^{-n}\right)} f(t) d t, & n \leqslant j \leqslant n+m .\end{cases}
$$

For $\nu=0$, we set

$$
r_{n+m, n}^{*}(z, f, 0)=r_{n+m, n}(z, f) .
$$

On using (1.3), an integral representation of $r_{n+m, n}^{*}(z, f, \nu), \nu \geqslant 1$, is found to be

$$
r_{n+m, n}^{*}(z, f, \nu)=\frac{1}{2 \pi i} \int_{\Gamma} \frac{f(t) F_{\nu}^{*}(t, \sigma)}{\left(z^{n}-\sigma^{n}\right)(t-z)} \sum_{j=1}^{3} A_{j}(t, z) K_{j}(t, \sigma) d t
$$

For a fixed integer $\ell \geqslant 1$, we set

$$
W_{n+m, n}^{*}(z, f, \ell):=\sum_{\nu=0}^{\ell-1} r_{n+m, n}^{*}(z, f, \nu)
$$

With the above notation, we can now prove

Theorem 2. Let $m \geqslant-1, q \geqslant 2$, and $\ell \geqslant 1$ be three fixed integers and $\sigma>1$. If $f \in A_{\rho}, 1<\rho<\infty$, then

$$
\lim _{n \rightarrow \infty}\left\{R_{n+m, n}^{*}(z, f)-W_{n+m, n}^{*}(z, f, \ell)\right\}=0, \begin{cases}|z|<\rho^{\ell q+1} & \text { if } \sigma \geqslant \rho^{\ell q+1} \\ |z| \neq \sigma & \text { if } \sigma<\rho^{\ell q+1}\end{cases}
$$

the convergence being uniform and geometric on any compact subset of the regions defined above. Moreover, the result is sharp.

Proof: As in (2.8), we use the relations (3.2), (3.7) and (3.8) to obtain

$$
\begin{aligned}
& R_{n+m, n}^{*}(z, f)-W_{n+m, n}^{*}(z, f, \ell) \\
& =\frac{1}{2 \pi i} \int_{\Gamma} \frac{\gamma_{\ell}(t, \sigma) f(t)}{\left(z^{n}-\sigma^{n}\right)(t-z)} \sum_{j=1}^{3} A_{j}(t, z) K_{j}(t, \sigma) d t
\end{aligned}
$$


where $\gamma_{\ell}(t, \sigma):=\sum_{s=0}^{\infty} \sum_{\lambda=1}^{q} F_{s q+\lambda}^{*}(t, \sigma)-\sum_{\lambda=1}^{\ell-1} F_{\lambda}^{*}(t, \sigma)$ and $\Gamma$ is the circle $|t|=\rho_{1}$ with $1<\rho_{1}<\rho$. If we write $\ell-1:=a q+b$ with $a \geqslant 0,0 \leqslant b \leqslant q-1$, then we have

$$
\begin{aligned}
\gamma_{\ell}(t, \sigma) & =\sum_{\cdot=0}^{\infty} \sum_{\lambda=1}^{q} F_{* q+\lambda}^{*}(t, \sigma)-\sum_{s=0}^{a-1} \sum_{\lambda=1}^{q} F_{s q+\lambda}^{*}(t, \sigma)-\sum_{\lambda=1}^{b} F_{a q+\lambda}(t, \sigma) \\
& =\sum_{s=a+1}^{\infty} \sum_{\lambda=1}^{q} F_{s q+\lambda}^{*}(t, \sigma)+\sum_{\lambda=b+1}^{q} F_{a q+\lambda}^{*}(t, \sigma) .
\end{aligned}
$$

that is,

$$
\gamma_{\ell}(t, \sigma)=\sum_{\cdot=1}^{\infty} \sum_{\lambda=1}^{q} F_{(s+a) q+\lambda}^{*}(t, \sigma)+\sum_{\lambda=b+1}^{q} F_{a q+\lambda}^{*}(t, \sigma) .
$$

Substituting the value of $F_{a q+\lambda}^{*}(t, \sigma)$ from (3.3), we can write

$$
\begin{aligned}
& \sum_{\lambda=b+1}^{q} F_{a q+\lambda}^{*}(t, \sigma) \\
& =\left(t^{n}-\sigma^{-n}\right)^{-a q^{2}} \sum_{\lambda=b+1}^{q}\left(t^{n}-\sigma^{-n}\right)^{-\lambda q} \\
& \quad \times\left\{1+\sum_{j=1}^{a}\left(\begin{array}{c}
(a-j) q^{2}+\lambda q+j-1 \\
j
\end{array}\right) \frac{\left(-\sigma^{-n}\right)^{j}}{\left(t^{n}-\sigma^{-n}\right)^{\left(1-q^{2}\right) j}}\right\} .
\end{aligned}
$$

If $\sigma \geqslant \rho^{\ell q+1}$ and $|t|=\rho_{1}$, it is easy to see that

$$
\begin{aligned}
& \left|\sum_{\lambda=b+1}^{q} F_{a q+\lambda}^{*}(t, \sigma)\right| \\
& \leqslant(q-b)\left(\rho_{1}^{n}-\sigma^{-n}\right)^{-(a q+b+1) q} \\
& \quad \times\left\{1+\frac{\left(\rho_{1}^{n}-\sigma^{-n}\right)^{q^{2}-1}}{\rho^{\ell q n}} \sum_{j=1}^{a}\left(\begin{array}{c}
(a-j) q^{2}+\lambda q+j-1 \\
j
\end{array}\right) \rho^{-j n}\right\},
\end{aligned}
$$

for all $n$ sufficiently large. Since $a q+b+1=: \ell$, we obtain

$$
\sum_{\lambda=b+1}^{q} F_{a q+\lambda}^{*}(t, \sigma)=O\left(\rho_{1}^{-\ell q n}\right) .
$$

It remains to estimate the double summation on the right side of (3.11). For this purpose, we set

$$
h(\nu, \mu)=\left(\begin{array}{c}
\mu q+(a+1) q^{2}+\nu-2 \\
\nu-1
\end{array}\right) \frac{\left(-\sigma^{-n}\right)^{\nu-1}}{\left(t^{n}-\sigma^{-n}\right)^{\mu q+(a+1) q^{2}+\nu-1}} .
$$


It remains to estimate the double summation on the right side of (3.11). For this purpose, we set

$$
h(\nu, \mu)=\left(\begin{array}{c}
\mu q+(a+1) q^{2}+\nu-2 \\
\nu-1
\end{array}\right) \frac{\left(-\sigma^{-n}\right)^{\nu-1}}{\left(t^{n}-\sigma^{-n}\right)^{\mu q+(a+1) q^{2}+\nu-1}} .
$$

Then using (3.3), we can rewrite

$$
\sum_{s=1}^{\infty} \sum_{\lambda=1}^{q} F_{(\bullet+a) q+\lambda}^{*}(t, \sigma)=I_{1}+I_{2}
$$

where

$$
\left\{\begin{array}{l}
I_{1}:=\sum_{s=1}^{\infty} \sum_{\lambda=1}^{q} \sum_{j=1}^{j} h(j,(s-j) q+\lambda), \\
I_{2}:=\sum_{s=1}^{\infty} \sum_{\lambda=1}^{\infty} \sum_{j=s+1}^{s+q+1} h(j,(s-j) q+\lambda) .
\end{array}\right.
$$

Recalling the identity (3.1), we obtain

$$
\begin{aligned}
& \sum_{\cdot=1}^{\infty} \sum_{\lambda=1}^{\infty} h(s, \lambda) \\
& =\frac{1}{\left(t^{n}-\sigma^{-n}\right)^{(a+1) q^{2}}} \sum_{\lambda=1}^{\infty} \frac{1}{\left(t^{n}-\sigma^{-n}\right)^{\lambda q}} \\
& \quad \times \sum_{\cdot=1}^{\infty}\left(\begin{array}{c}
\lambda q+(a+1) q^{2}+s-2 \\
s-1
\end{array}\right)\left(\frac{-\sigma^{-n}}{t^{n}-\sigma^{-n}}\right)^{s-1} \\
& =\left(t^{n}-\sigma^{-n}\right)^{-(a+1) q^{2}} \sum_{\lambda=1}^{\infty}\left(t^{n}-\sigma^{-n}\right)^{-\lambda q}\left(1+\frac{\sigma^{-n}}{t^{n}-\sigma^{-n}}\right)^{-\left(\lambda q+(a+1) q^{2}\right)}
\end{aligned}
$$

so that

$$
I_{1}=t^{-(a+1) n q^{2}}\left(t^{q n}-1\right)^{-1}=O\left(\rho_{1}^{-(a+1) n q^{2}-q n}\right) .
$$

Further, we notice that

$$
I_{2}:=\sum_{s=1}^{\infty} \sum_{\lambda=1}^{q} \sum_{j=1}^{q+1} h(j+s,-j q+\lambda)
$$

where in view of (3.13)

$$
\begin{aligned}
& h(j+s,-j q+\lambda) \\
& =\left(\begin{array}{c}
(-j q+\lambda) q+(a+1) q^{2}+j+s-2 \\
(-j q+\lambda) q+(a+1) q^{2}-1
\end{array}\right) \frac{\left(-\sigma^{-n}\right)^{j-1+s}}{\left(t^{n}-\sigma^{-n}\right)^{(a+1-j) q^{2}+\lambda q+j+-1}} .
\end{aligned}
$$


Since $d(s):=\sum_{\lambda=1}^{q} \sum_{j=1}^{a+1}\left(\begin{array}{c}(\lambda-j q) q+(a+1) q^{2}+j+-2 \\ (\lambda-j q) q+(a+1) q^{2}-1\end{array}\right)$ is a polynomial in $s$ of degree at most $(a+1) q^{2}-1$, it follows that for all $n$ sufficiently large, the function $\sum_{s=1}^{\infty} d(s)\left(t^{n}-\sigma^{-n}\right)^{-\cdot}$ is analytic for $|t|>1([4]$, Lemma 2). Thus, there is a constant $c_{0}$ independent of $n$ such that

$$
\left|\sum_{s=1}^{\infty} d(s)\left(t^{n}-\sigma^{-n}\right)^{-\cdot}\right| \leqslant c_{0}
$$

Since $\sigma \geqslant \rho^{\ell q+1}$ and $|t|=\rho_{1}$, it follows from (3.16) and (3.17) after some elementary algebra that for sufficiently large $n$

$$
\left|I_{2}\right| \leqslant c_{0} \rho^{-n(\ell q+1)}\left(\rho_{1}^{n}-\sigma^{-n}\right)^{-a q^{2}-q} .
$$

Recall that $\ell q:=(a q+b+1) q \leqslant(a+1) q^{2}$. Therefore, combining (3.11), (3.12), (3.14) and (3.18), we observe that

$$
\left|\gamma_{\ell}(t, \sigma)\right| \leqslant \frac{c^{*}}{\rho_{1}^{n \ell q}}, \text { for all sufficiently large } n,
$$

where $c^{*}$ is a constant independent of $n$. Using (3.10) and (3.19), an analysis of the kernels $A_{j}(t, z) K_{j}(t, \sigma), j=1,2,3$, shows that

$$
\varlimsup_{n \rightarrow \infty}\left\{\max _{|z|=\tau}\left|R_{n+m, n}^{*}(z, f)-W_{n+m, n}^{*}(z, f, \ell)\right|\right\}^{1 / n} \leqslant \frac{\tau}{\rho_{1}^{\ell q+1}} .
$$

When $\sigma<\rho^{\ell q+1}$, a similar analysis of $\gamma_{\ell}(t, \sigma)$ and $A_{j}(t, z) \cdot K_{j}(t, \sigma)$ gives us

$$
\lim _{n \rightarrow \infty}\left\{R_{n+m, n}^{*}(z, f)-W_{n+m, n}^{*}(z, f, \ell)\right\}=0,
$$

for all $z$ with $|z| \neq \sigma$.

The sharpness of the result can be seen by considering

$$
\widehat{f}(z)=\left(z-\rho e^{i \theta}\right)^{-1} \text { where } 0 \leqslant \theta \leqslant 2 \pi .
$$

REMARK 2. Theorems 1 and 2 are also valid when $q=1$ and $m=-1$ (see [2], Remark 3.1). Therefore, a result of Saff-Sharma ([6], Theorem 3.1), under the condition $m=-1$, is a special case of Theorem 1 .

REMARK 3. If we fix $m=-1$ and let $\sigma \rightarrow \infty$ in either of Theorems 1 and 2 , we get an extension of Rivlin's result given in (1.7). This follows from the fact that (see (2.1), (3.5)) for all integers $n \geqslant 1, \nu \geqslant 0$,

$$
\lim _{\sigma \rightarrow \infty} \tilde{r}_{n-1, n}(z, f, \nu)=\lim _{\sigma \rightarrow \infty} r_{n-1, n}^{*}(z, f, \nu)=S_{n-1, \nu}(z, f),
$$

where $S_{n-1, \nu}(z, f)$ is described in (1.7). 


\section{REFERENCES}

[1] M.A. Bokhari, Equiconvergence of some interpolatory and best approximating processes, Ph.D. Thesis (University of Alberta, Edmonton, Canada, 1986).

[2] M.A. Bokhari, 'On certain sequences of least squares approximants', Bull. Austral. Math. Soc. 37 (1988), 415-422.

[3] M.A. Bokhari, 'Equiconvergence of some sequences of complex interpolating rational functions (Quantitative estimates and sharpness)', J. Approx. Theory 55 (1988), 205-219.

[4] A.S. Cavaretta Jr., A. Sharma and R.S. Varga, 'Interpolation in the roots of unity: An extension of a theorem of J.L. Walsh', Resultate Math. 3 (1981), 155-191.

[5] T.J. Rivlin, 'On Walsh equiconvergence', J. Approx. Theory 36 (1982), 334-345.

[6] E.B. Saff and A. Sharma, 'On equiconvergence of certain sequences of rational interpolants', in Proc. Rational Approximations and Interpolation: Lecture Notes in Math 1105, pp. 256-2711 (Springer-Verlag, Berlin, Heidelberg, New York, Tokyo, 1984).

[7] R.S. Varga, 'Topics in polynomial and rational approximation', in Seminaire de Math., pp. 69-93 (Superieures Les Presses de L'Univ. de Montreal, 1982).

[8] J.L. Walsh, Interpolation and approximation by rational functions in the complex domain (Amer. Math. Soc. Colloq. Publ. 20, Providence, R.I., 1969).

Department of Mathematical Sciences

King Fahd University of Petroleum and Minerals

Dhahran 31261

Saudi Arabia 